\title{
On MF-projective modules
}

\author{
Yusuf Alagöz (iD \\ Siirt University, Department of Mathematics, Siirt, Turkey
}

\begin{abstract}
In this paper, we study the left orthogonal class of max-flat modules which are the homological objects related to s-pure exact sequences of modules and module homomorphisms. Namely, a right module $A$ is called $M F$-projective if $\operatorname{Ext}_{R}^{1}(A, B)=0$ for any max-flat right $R$-module $B$, and $A$ is called strongly $M F$-projective if $\operatorname{Ext}_{R}^{i}(A, B)=0$ for all max-flat right $R$-modules $B$ and all $i \geq 1$. Firstly, we give some properties of $M F$-projective modules and SMF-projective modules. Then we introduce and study MF-projective dimensions for modules and rings. The relations between the introduced dimensions and other (classical) homological dimensions are discussed. We characterize some classes of rings such as perfect rings, $Q F$ rings and max-hereditary rings by $(S) M F$-projective modules. We also study the rings whose right ideals are MF-projective. Finally, we characterize the rings whose $M F$-projective modules are projective.
\end{abstract}

Mathematics Subject Classification (2020). 16D40, 16E10, 18G25

Keywords. (max-)flat modules, MF-projective modues, max-hereditary rings

\section{Introduction}

Throughout, $R$ will denote an associative ring with identity, and modules will be unital right $R$-modules, unless otherwise stated. As usual, we denote by $\mathfrak{M}_{R}(R \mathfrak{M})$ the category of right (left) $R$-modules. For a module $A, E(A), i d(A), p d(A)$ and $A^{+}$denote the injective hull, injective dimension, projective dimension and the character module $\operatorname{Hom}_{\mathbb{Z}}(A, \mathbb{Q} / \mathbb{Z})$ of $A$, respectively.

Let $\mathfrak{C}$ be a class of $R$-modules and $A$ be an $R$-module. A homomorphism $f: A \rightarrow C$ with $C \in \mathfrak{C}$ is called a $\mathfrak{C}$-preenvelope of $A$ if for any homomorphism $g: A \rightarrow D$ with $D \in \mathfrak{C}$, there is a homomorphism $h: C \rightarrow D$ such that $h f=g$ (see [8]). Moreover, if the only such $h$ are automorphisms of $C$ when $C=D$ and $g=f$, the $\mathfrak{C}$-preenvelope is called a $\mathfrak{C}$-envelope of $A$. Dually, we have the definitions of a $\mathfrak{C}$-precover and a $\mathfrak{C}$-cover. $\mathfrak{C}$-envelopes ( $\mathfrak{C}$-covers) may not exist in general, but if they exist, they are unique up to isomorphism. We will denote by $\mathfrak{C}^{\perp}=\left\{X: \operatorname{Ext}_{R}^{1}(C, X)=0\right.$ for all $\left.C \in \mathfrak{C}\right\}$ the right orthogonal class of $\mathfrak{C}$, and by ${ }^{\perp} \mathfrak{C}=\left\{X: \operatorname{Ext}_{R}^{1}(X, C)=0\right.$ for all $\left.C \in \mathfrak{C}\right\}$ the left orthogonal class of $\mathfrak{C}$. A pair $(\mathfrak{F}, \mathfrak{C})$ of classes of right $R$-modules is called a cotorsion theory (for the category of $R$-modules) if $\mathfrak{F}^{\perp}=\mathfrak{C}$ and ${ }^{\perp} \mathfrak{C}=\mathfrak{F}$. A cotorsion theory $(\mathfrak{F}, \mathfrak{C}$ ) is called perfect (complete) if every right $R$-module has a $\mathfrak{C}$-envelope and an $\mathfrak{F}$-cover (a special $\mathfrak{C}$-preenvelope and a special $\mathfrak{F}$-precover $)$. A cotorsion theory $(\mathfrak{F}, \mathfrak{C})$ is said to be

Email address: yusuf.alagoz@siirt.edu.tr

Received: 03.05.2020; Accepted: 23.08.2020 
hereditary if whenever $0 \rightarrow L^{\prime} \rightarrow L \rightarrow L^{\prime \prime} \rightarrow 0$ is exact with $L, L^{\prime \prime} \in \mathfrak{F}$, then $L^{\prime}$ is also in $\mathfrak{F}$ (see [9]). By [9], ( $\mathfrak{F}, \mathfrak{C}$ ) is hereditary if and only if whenever $0 \rightarrow C^{\prime} \rightarrow C \rightarrow C^{\prime \prime} \rightarrow 0$ is exact with $C, C^{\prime} \in \mathfrak{C}$, then $C^{\prime \prime}$ is also in $\mathfrak{C}$.

Since its development, the Cohn purity plays a significant role in module theory and homological algebra. One of the main reason is that, some significant homological objects such as, flat modules, cotorsion modules, absolutely pure modules and pure-injective modules arose from this notion of purity. Recall that, the submodule $A$ of $B$ is called s-pure submodule of $B$ [5] if $i \otimes 1_{S}: A \otimes S \rightarrow B \otimes S$ is a monomorphism for each simple left module $S$. Similarly, the submodule $A$ of $B$ is called neat submodule of $B$ if $\operatorname{Hom}(S, B) \rightarrow \operatorname{Hom}(S, B / A)$ is an epimorphism for each simple right module $S$. Unlike the generation of pure submodules, the notions of s-pure and neat submodules are not only inequivalent they are also incomparable. The equality of the notions of s-pure and neat submodules is considered in [12], which is hold over the commutative domains whose maximal ideals are invertible, and these domains termed as $N$-domains. In [6], S. Crivei proved that if the ring is commutative and the maximal ideals are principal, then the notions s-pure and neat submodules coincide. Recently, the commutative rings with this property are completely characterized in [19, Theorem 3.7]. These are exactly the commutative rings whose maximal ideals are finitely generated and locally principal.

A left $R$-module $A$ is called max-injective if for the inclusion map $i: I \rightarrow R$ with $I$ maximal left ideal, and any homomorphism $f: I \rightarrow A$ there exist a homomorphism $g: R \rightarrow A$ such that $g i=f$, or equivalently $\operatorname{Ext}_{R}^{1}(R / I, A)=0$ for any maximal left ideal $I$. A ring $R$ is said to be left max-injective if $\mathrm{R}$ is max-injective as a left $R$-module [26]. As observed by Crivei in [6, Theorem 3.4], a left $R$-module $A$ is max-injective if and only if $A$ is a neat submodule of every module containing it. A right $R$-module $A$ is called max-flat if $\operatorname{Tor}_{1}^{R}(A, R / I)=0$ for any maximal left ideal $I$ of $R$ (see [25]). A right $R$-module $A$ is max-flat if and only if $A^{+}$is max-injective by the isomorphism $\operatorname{Ext}_{R}^{1}\left(R / I, A^{+}\right) \cong\left(\operatorname{Tor}_{1}^{R}(A, R / I)\right)^{+}$for any maximal left ideal $I$ of $R$. Indeed, we show in Lemma 4.1 that, a right $R$-module $A$ is max-flat if and only if any short exact sequence ending with $A$ is s-pure.

So far, s-pure and neat submodules and homological objects related to s-pure and neatexact sequences are studied by many authors (see, $[3,5-7,12-14,19,26,27]$ ).

The main purpose of this paper is to continue the study and investigation of the homological objects related to s-pure and neat short exact sequences. Namely, we have studied max-flat modules and left orthogonal class of max-flat modules.

Along the way, the concepts of $M F$-projective and strongly $M F$-projective modules are first introduced in section 2. Several elementary properties of $M F$-projective and $S M F$ projective modules are obtained in this section. We prove that a right $R$-module $A$ is $M F$-projective if and only if $A$ is a cokernel of a max-flat preenvelope $f: C \rightarrow B$ with $B$ projective. It is shown that a ring $R$ is right perfect if and only if all max-flat right $R$-modules are $(S) M F$-projective. It is also proven that $R$ is a $Q F$ ring if and only if every right $R$-module is $(S) M F$-projective.

In section 3 of this article, we define and discuss $M F$-projective dimensions for modules and rings. For a right $R$-module $A$, the $M F$-projective dimension $m f p d(A)$ of $A$ is defined to be the smallest integer $n \geq 0$ such that $\operatorname{Ext}_{R}^{n+i}(A, B)=0$ for any max-flat right $R$ module $B$ and any integer $i \geq 1$. If no such $n$ exists, set $m f p d(A)=\infty$. Put $\operatorname{rmfpD}(R)=$ $\sup \{m f p d(A): A$ is a right $R$-module $\}$, and call $\operatorname{rmfpD}(R)$ the right MF-projective dimension of $R$. It is proven that $\operatorname{rmf} D(R) \leq n$ if and only if $i d(A) \leq n$ for all maxflat right $R$-modules $A$. Certain characterizations of QF rings in terms of $M F$-projective modules are also obtained. We characterize the rings whose simple right $R$-modules are $M F$-projective. We also introduce the notion of right $M F$-hereditary rings, and then give some characterizations of such rings. It is shown that a ring $R$ is right $M F$-hereditary if 
and only if every submodule of an $M F$-projective right $R$-module is $M F$-projective if and only if $\operatorname{rmf} D(R) \leq 1$ if and only if $i d(A) \leq 1$ for all max-flat right $R$-modules $A$.

In section 4 , we study max-flat preenvelopes which are epimorphisms. We first consider the commutative rings whose maximal ideals are finitely generated and locally principal over which neat-flat modules and max-flat modules coincide. By using this result, over a commutative ring whose maximal ideals are finitely generated and locally principal it is proven that the following are equivalent: (1) $\mathrm{R}$ is max-hereditary; (2) every (simple) $R$ module has an epic max-flat preenvelope; (3) every simple $R$-module has an epic projective preenvelope; (4) every (finitely presented) $M F$-projective module is projective; (5) $R$ is a $P S$ ring.

\section{Left orthogonal class of max-flat modules}

We begin with the following definition.

Definition 2.1. A right module $A$ is called $M F$-projective if $\operatorname{Ext}_{R}^{1}(A, B)=0$ for any max-flat right $R$-module $B . A$ is said to be strongly $M F$-projective ( $S M F$-projective for short) if $\operatorname{Ext}_{R}^{i}(A, B)=0$ for all max-flat right $R$-modules $B$ and all $i \geq 1$.

Recall that a ring $R$ is said to be a left $C$-ring if $\operatorname{Soc}(R / I) \neq 0$ for every proper essential left ideal $I$ of $R$. Right perfect rings, left semiartinian rings are well known examples of left $C$-rings $([4,10.10])$.

Remark 2.2. (1) Projective modules are clearly $(S) M F$-projective, but the converse need not to be true in general. For example, let $R$ be a local $Q F$ ring $R=k[X] /\left(X^{2}\right)$, where $k$ is a field, and $\bar{X}$ denotes the residue class of $X$ in $R$. Then every right $R$-module is (S) $M F$-projective by Proposition 2.11, so is the ideal $\bar{X}$, in particular. However $\bar{X}$ is not projective, because $\bar{X}^{2}=0$ implies that $\bar{X}$ is not a free ideal in the local ring $R$.

(2) In [11], Fu et al. defined and discussed copure-projective modules. A right module $A$ is called copure-projective provided that $\operatorname{Ext}_{R}^{1}(A, B)=0$ for any flat right module $B$. Since every flat right module is max-flat, every $M F$-projective right module is copureprojective. For the converse, let $R$ be a left $C$-ring. It is shown in [24, Lemma 4] that every max-injective left module is injective, so in this case, every max-flat right module is flat. Thus every copure-projective right module is $M F$-projective.

Recall that the class of max-flat modules is closed under extensions, direct sums, direct summands by [27, Proposition 2.4(2)]. Moreover it is closed under pure submodules and pure quotients by the following lemma.

Lemma 2.3. (1) The class of max-flat modules is closed under pure submodules and pure quotients.

(2) The class of MF-projective modules is closed under extensions, direct sums and direct summands.

Proof. (1) Consider the pure exact sequence of right $R$-modules $0 \rightarrow B \rightarrow A \rightarrow A / B \rightarrow 0$ with $A$ max-flat. Since $0 \rightarrow(A / B)^{+} \rightarrow A^{+} \rightarrow B^{+} \rightarrow 0$ splits and $A^{+}$is max-injective, $B^{+}$and $(A / B)^{+}$is max-injective. Hence $B$ and $A / B$ is max-flat.

(2) The class of $M F$-projective modules is closed under extensions by using the functor $\operatorname{Ext}_{R}^{1}(-, F)$ for any max-flat module $F$. Also, it is closed under direct sums and direct summands by using the isomorphism $\operatorname{Ext}_{R}^{1}\left(\oplus_{i \in I} A_{i}, F\right) \cong \prod_{i \in I} \operatorname{Ext}_{R}^{1}\left(A_{i}, F\right)$ for any maxflat module $F$ and a family of modules $\left(A_{i}\right)_{i \in I}$ by [23, Theorem 7.13].

Recall that a ring $R$ is called left max-hereditary if every maximal left ideal is projective (see [1]). This is equivalent to saying that every factor of a max-injective left $R$-module is max-injective (see [1, Proposition 1.2]). A ring $R$ is called a left $S F$-ring if each simple left $R$-module is flat (see [22]). The following example shows that a left max-hereditary ring does not need to be left SF-ring. 
Example 2.4. Assume that $R$ is a left Noetherian left hereditary ring that is not semisimple. Thus every left ideal of $R$ is projective, and so $R$ is left max-hereditary. But $R$ is not a left $S F$-ring. Otherwise, since $R$ is left Noetherian, every simple left $R$-module is finitely presented. If $R$ was a left SF-ring, then every simple left $R$-module would be projective by [23, Corollary 3.58], whence $R$ would be semisimple, a contradiction.

We shall now give a condition for the converse of Remark 2.2(1).

Proposition 2.5. Let $R$ be a left max-hereditary ring or a left SF-ring. Then the followings are equivalent for a module $A$.

(1) A is projective.

(2) A is SMF-projective.

(3) A is MF-projective.

Proof. We know that $(1) \Rightarrow(2) \Rightarrow(3)$ is always true.

(a) First, assume that $R$ is a left max-hereditary ring.

(3) $\Rightarrow(1)$ Let $A$ be an $M F$-projective right module. Then there is an exact sequence $0 \rightarrow C \rightarrow B \rightarrow A \rightarrow 0$ with $B$ projective. Then this exact sequence induces the exactness of $0 \rightarrow A^{+} \rightarrow B^{+} \rightarrow C^{+} \rightarrow 0$. Since $B^{+}$is injective, $C^{+}$is max-injective by [1, Proposition 1.2] and so $C$ is max-flat. Thus, $\operatorname{Ext}_{R}^{1}(A, C)=0$, that is, $0 \rightarrow C \rightarrow B \rightarrow A \rightarrow 0$ splits. It follows that $A$ is projective.

(b) Now, assume that $R$ is a left $S F$-ring.

(3) $\Rightarrow(1)$ Let $A$ be an $M F$-projective right module. Then there is an exact sequence $0 \rightarrow C \rightarrow B \rightarrow A \rightarrow 0$ with $B$ projective. Since $R$ is a left $S F$-ring, $\operatorname{Tor}_{1}^{R}(C, R / I)=0$ for any maximal left ideal $I$ of $R$, and so $C$ is max-flat. Thus, $\operatorname{Ext}_{R}^{1}(A, C)=0$, that is, $0 \rightarrow C \rightarrow B \rightarrow A \rightarrow 0$ splits. It follows that $A$ is projective.

By definitions, every $S M F$-projective module is $M F$-projective. For the converse we have the following condition.

Proposition 2.6. Let $R$ be a ring and $A$ an $M F$-projective right $R$-module. Then $A$ is $S M F$-projective if and only if for any exact sequence $0 \rightarrow C \rightarrow B \rightarrow A \rightarrow 0$ of right $R$-modules with $B$ projective, $C$ is $S M F$-projective.

Proof. Let $0 \rightarrow C \rightarrow B \rightarrow A \rightarrow 0$ be an exact sequence of right $R$-modules with $B$ projective. If $A$ is $S M F$-projective, then $\operatorname{Ext}_{R}^{i}(C, F) \cong \operatorname{Ext}_{R}^{i+1}(A, F)=0$ for any max-flat right $R$-module $F$ and $i \geq 1$. So $C$ is $S M F$-projective. Conversely, if $C$ is $S M F$-projective, then $\operatorname{Ext}_{R}^{i}(A, F) \cong \operatorname{Ext}_{R}^{i-1}(C, F)=0$ for any max-flat right $R$-module $F$ and $i \geq 2$. But $\operatorname{Ext}_{R}^{1}(A, F)=0$ by hypothesis, and so $A$ is $S M F$-projective.

The following proposition gives some characterizations of $M F$-projective modules in terms of max-flat preenvelopes.

Proposition 2.7. The following are equivalent for a right $R$-module $A$.

(1) A is MF-projective.

(2) $A$ is projective with respect to every exact sequence $0 \rightarrow K \rightarrow T \rightarrow L \rightarrow 0$ with $K$ max-flat.

(3) For every exact sequence $0 \rightarrow C \rightarrow B \rightarrow A \rightarrow 0$, with $B$ max-flat, $C \rightarrow B$ is a max-flat preenvelope of $C$.

(4) $A$ is a cokernel of a max-flat preenvelope $C \rightarrow B$ with $B$ projective.

Proof. (1) $\Rightarrow(2)$ and (1) $\Rightarrow(3)$ are trivial.

$(2) \Rightarrow(1)$ Let $B$ be a max-flat right $R$-module. The exactness of the sequence $0 \rightarrow B \rightarrow$ $E(B) \rightarrow E(B) / B \rightarrow 0$ induces the exact sequence $\operatorname{Hom}(A, E(B)) \rightarrow \operatorname{Hom}(A, E(B) / B) \rightarrow$ $\operatorname{Ext}_{R}^{1}(A, B) \rightarrow 0$. Since $\operatorname{Hom}(A, E(B)) \rightarrow \operatorname{Hom}(A, E(B) / B)$ is epic by $(2), \operatorname{Ext}_{R}^{1}(A, B)=$ 0 . So $A$ is $M F$-projective. 
(3) $\Rightarrow$ (4) Since there is an exact sequence $0 \rightarrow C \rightarrow P \rightarrow A \rightarrow 0$ with $P$ projective, (4) follows from (3).

$(4) \Rightarrow(1)$ Let $A$ be a cokernel of a max-flat preenvelope $f: C \rightarrow B$ with $B$ projective. Then, there is an exact sequence $0 \rightarrow D \rightarrow B \rightarrow A \rightarrow 0$ with $D=\operatorname{Im}(f)$. For each max-flat right $R$-module $F$, the sequence $\operatorname{Hom}(B, F) \rightarrow \operatorname{Hom}(D, F) \rightarrow \operatorname{Ext}_{R}^{1}(A, F) \rightarrow 0$

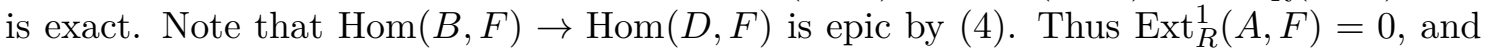
so $A$ is $M F$-projective.

Now we characterize $M F$-projective modules over a commutative ring.

Proposition 2.8. The following statements are equivalent for a commutative ring $R$ and an $R$-module $A$.

(1) $A$ is $M F$-projective.

(2) $P \otimes_{R} A$ is $M F$-projective for any projective $R$-module $P$.

(3) $\operatorname{Hom}(P, A)$ is $M F$-projective for any finitely generated projective $R$-module $P$.

Proof. $(1) \Rightarrow(2)$ Let $P$ be a projective $R$-module and consider by [23, Exercise 9.20] the isomorphism $\operatorname{Ext}_{R}^{1}\left(P \otimes_{R} A, B\right) \cong \operatorname{Hom}\left(P, \operatorname{Ext}_{R}^{1}(A, B)\right)$. For any max-flat $R$-module $B$, we have $\operatorname{Ext}_{R}^{1}(A, B)=0$ since $A$ is $M F$-projective. This says that $\operatorname{Ext}_{R}^{1}\left(P \otimes_{R} A, B\right)=0$. Thus $P \otimes_{R} A$ is $M F$-projective.

$(1) \Rightarrow(3)$ Let $P$ be a finitely generated projective $R$-module. By using [23, Lemma 3.59] and mimicking the proof of [23, Theorem 9.51], we have the isomorphism $P \otimes_{R}$ $\operatorname{Ext}_{R}^{1}(A, B) \cong \operatorname{Ext}_{R}^{1}(\operatorname{Hom}(P, A), B)$. Since $A$ is $M F$-projective, $\operatorname{Ext}_{R}^{1}(A, B)=0$ for any max-flat $R$-module $B$. This says that $\operatorname{Ext}_{R}^{1}(\operatorname{Hom}(P, A), B)=0$, and so $\operatorname{Hom}(P, A)$ is $M F$-projective.

$(2) \Rightarrow(1)$ and $(3) \Rightarrow(1)$ are clear by letting $P=R$.

A ring $R$ is called left max-coherent if every maximal left ideal is finitely presented. A right $R$-module $A$ is called $M I$-flat if $\operatorname{Tor}_{1}^{R}(A, B)=0$ for any max-injective left $R$-module $B$ (see [27]). These modules were discovered when studying max-flat preenvelopes.

Proposition 2.9. Let $R$ be a left max-coherent ring. Then:

(1) Every MF-projective right $R$-module is $M I$-flat.

(2) Every finitely presented $M I$-flat right $R$-module is $M F$-projective.

Proof. (1) Let $A$ be an $M F$-projective right $R$-module. For any max-injective left $R$ module $E, E^{+}$is max-flat by [27, Theorem 2.3], and hence $\operatorname{Ext}_{R}^{1}\left(A, E^{+}\right)=0$. Thus from the standard isomorphism $\operatorname{Ext}_{R}^{1}\left(A, E^{+}\right) \cong\left(\operatorname{Tor}_{1}^{R}(A, E)\right)^{+}$in [8, Theorem 3.2.1], we have $\operatorname{Tor}_{1}^{R}(A, E)=0$. So $A$ is $M I$-flat.

(2) Let $A$ be a finitely presented $M I$-flat right $R$-module. Then $A$ is the cokernel of a max-flat preenvelope $g: C \rightarrow B$ with $B$ projective by [27, Proposition 3.7(2)]. Hence, $A$ is $M F$-projective by Proposition 2.7 .

It is well known that $R$ is a right perfect ring if and only if every flat right $R$-module is projective. The converse of Proposition 2.9(1) characterizes the right perfect rings over a left max-coherent ring.

Theorem 2.10. Let $R$ be a ring. Then the followings are equivalent.

(1) $R$ is right perfect.

(2) All max-flat right $R$-modules are projective.

(3) All max-flat right $R$-modules are $S M F$-projective.

(4) All max-flat right $R$-modules are $M F$-projective.

(5) All flat right $R$-modules are $M F$-projective.

Also, if $R$ is a left max-coherent ring, then the above conditions are equivalent to:

(6) All MI-flat right $R$-modules are MF-projective. 
Proof. $(2) \Rightarrow(3) \Rightarrow(4) \Rightarrow(5)$ and $(6) \Rightarrow(5)$ are clear.

$(1) \Rightarrow(2)$ Let $A$ be any max-flat right $R$-module. Then $A^{+}$is max-injective. Since $R$ is a left $C$-ring, $A^{+}$is injective by [24, Lemma 4], whence $A$ is flat. By the perfectness of $R, A$ is projective.

$(5) \Rightarrow(1)$ Let $A$ be a flat right $R$-module. There is an exact sequence $0 \rightarrow B \rightarrow$ $P \rightarrow A \rightarrow 0$ with $P$ projective. Note that, by the flatness of $A, B$ is flat. Since $A$ is $M F$-projective by (5), $\operatorname{Ext}_{R}^{1}(A, B)=0$. So $0 \rightarrow B \rightarrow P \rightarrow A \rightarrow 0$ splits, whence $A$ is projective.

$(1) \Rightarrow(6)$ Let $A$ be an $M I$-flat right $R$-module and $F$ a max-flat right $R$-module. Since $R$ is a left $C$-ring, $F^{+}$is injective by [1, Corollary 1.1], and so $F$ is flat. Also, since $R$ is a left max-coherent ring, $R$ is left coherent by [1, Corollary 1.1]. Thus, right perfectness of $R$ gives from [16, Proposition 1.4] that pure injectivity of $F$. But $F$ is a pure submodule of $F^{++}$, so $F$ is a direct summand of a max-flat right $R$-module $F^{++}$. Because $F^{+}$is max-injective, $\operatorname{Ext}_{R}^{1}\left(A, F^{++}\right) \cong\left(\operatorname{Tor}_{1}^{R}\left(A, F^{+}\right)\right)^{+}=0$. Therefore $\operatorname{Ext}_{R}^{1}(A, F)=0$. So, $A$ is $M F$-projective.

Recall that $R$ is said to be a $Q F$-ring if $R$ is left Noetherian and left self-injective, or equivalently $R$ is right artinian and right self-injective. By a well-known result of Faith and Walker [10], $\mathrm{R}$ is $\mathrm{QF}$ if and only if every projective right $R$-module is injective. In the following result, we give a new characterization of a $Q F$ ring.

Proposition 2.11. $R$ is a $Q F$ ring if and only if every right $R$-module is $(S) M F$ projective.

Proof. Let $A$ be a right $R$-module and $B$ a max-flat right $R$-module. Since $R$ is right artinian, $R$ is right perfect, and so $B$ is projective by Theorem 2.10. Thus $B$ is an injective right $R$-module by the hypoyhesis. This means that $\operatorname{Ext}_{R}^{i+1}(A, B)=0$ for any max-flat right $R$-module $B$ and any $i \geq 0$. Hence $A$ is $(S) M F$-projective. Conversely, let $A$ be a projective right $R$-module. Since $A$ is max-flat, by the hypoyhesis $\operatorname{Ext}_{R}^{i+1}(B, A)=0$ for any right $R$-module $B$ and any $i \geq 0$. So $A$ is injective, whence $R$ is a $Q F$-ring.

In the following, we characterize when every simple right module is $M F$-projective.

Lemma 2.12. Every simple right $R$-module is $M F$-projective if and only if every max-flat right $R$-module is max-injective.

Proof. Let $A$ be a max-flat right $R$-module. Then by the hypothesis, $\operatorname{Ext}_{R}^{1}(R / I, A)=0$ for any maximal right ideal $I$ of $R$. It follows that $A$ is max-injective. Conversely, let $S$ be a simple right $R$-module. For any max-flat right $R$-module $A, A$ is max-injective. Thus $\operatorname{Ext}_{R}^{1}(S, A)=0$, whence $S$ is $M F$-projective.

In general, a left SF-ring does not need to be a semisimple ring. The fact that every simple right (left) $R$-module is projective if and only if $R$ is semisimple together with Proposition 2.5 and Lemma 2.12 gives rise the following corollary.

Corollary 2.13. Let $R$ be a ring. The followings are equivalent.

(1) $R$ is a semisimple ring.

(2) $R$ is a left max-coherent left $S F$-ring.

(3) $R$ is a left max-hereditary ring and every simple right $R$-module is $M F$-projective.

(4) $R$ is a left max-hereditary ring and every max-flat right $R$-module is max-injective. 


\section{MF-projective dimensions}

In this section we investigate the MF-projective dimension of modules. We begin with the following definition.

Definition 3.1. Let $R$ be a ring. For a right $R$-module $A$, let $m f p d(A)$ denote the smallest integer $n \geq 0$ such that $\operatorname{Ext}_{R}^{n+i}(A, B)=0$ for any max-flat right $R$-module $B$ and any integer $i \geq 1$, and call $\operatorname{mfpd}(A)$ the $M F$-projective dimension of $A$. If no such $n$ exists, set $m f p d(A)=\infty$.

Put $\operatorname{rmfp} D(R)=\sup \{m f p d(A): A$ is a right $R$-module $\}$, and call $r m f p D(R)$ the right $M F$-projective dimension of $R$. Similarly we have $\operatorname{lmfpD}(R)$.

The following remark follows from definitions and Proposition 2.11.

Remark 3.2. (1) A module $A$ is $S M F$-projective if and only if $m f p d(A)=0$.

(2) A ring $R$ is a $Q F$-ring if and only if $\operatorname{rmfp} D(R)=0$.

The copure projective dimension $\operatorname{cpd}(A)$ of an $R$-module $A$ is defined in [11] as the smallest integer $n \geq 0$ such that $\operatorname{Ext}_{R}^{n+i}(A, B)=0$ for any flat right $R$-module $B$ and any $i \geq 1$. The right copure projective dimension of a ring $R$ is defined as $\operatorname{rcp} D(R)=$ $\sup \{\operatorname{cpd}(A) \mid A$ is a right $\mathrm{R}$-module $\}$. By the following proposition, we have the relation with right copure projective dimension of rings.

Proposition 3.3. Let $R$ be a ring. Then $r m f p D(R) \leq \operatorname{rcp} D(R)$. Moreover, if $r c p D(R)<$ $\infty$, then $\operatorname{rmfp} D(R)=\operatorname{rcp} D(R)$.

Proof. It is clear that $\operatorname{rmfp} D(R) \leq \operatorname{rcp} D(R)$, since any flat right $R$-module is max-flat. Now suppose that $\operatorname{rmfp} D(R)=n<\infty$. Let $A$ be a right $R$-module with $\operatorname{cpd}(A)=k<\infty$. Suppose $k>n$. For any flat right $R$-module $B$, consider the short exact sequence $0 \rightarrow$ $C \rightarrow P \rightarrow B \rightarrow 0$ with $P$ projective. Since $B$ and $P$ are flat, $C$ is flat by [17, Corollary 4.86]. So we get an exact sequence $\operatorname{Ext}_{R}^{k}(A, P) \rightarrow \operatorname{Ext}_{R}^{k}(A, B) \rightarrow \operatorname{Ext}_{R}^{k+1}(A, C)$. Since $\operatorname{rmfp} D(R)=n<k, \operatorname{Ext}_{R}^{k}(A, P)=0$. Also since $\operatorname{cpd}(A)=k, \operatorname{Ext}_{R}^{k+1}(A, C)=0$. Then $\operatorname{Ext}_{R}^{k}(A, B)=0$, whence $\operatorname{cpd}(A)<k$, a contradiction. Thus $k \leq n$, and $\operatorname{rcp} D(R) \leq$ $\operatorname{rmfpD}(R)$.

It is clear that $r m f p D(R) \leq r D(R)$, where $r D(R)$ denote the right global dimension of $R$. In general, $r m f p D(R) \neq r D(R)$. For example, let $R$ be a $Q F$ ring with $r D(R) \neq 0$ (e.g. $R=\mathbb{Z} / 4 \mathbb{Z}$ ), then $\operatorname{rmfpD}(R)=0$. The next corollary is due to Fu et al. [11, Corollary 4.4].

Corollary 3.4. Let $R$ be a ring with $r D(R)<\infty$. Then $r m f p D(R)=\operatorname{rcp} D(R)=r D(R)$.

From now on, for the class of $S M F$-projective right $R$-modules we write $\mathscr{S} \mathscr{M} \mathscr{F}$.

Lemma 3.5. ( $\left.\mathscr{S} \mathscr{M} \mathscr{F}, \mathscr{S} \mathscr{M} \mathscr{F}^{\perp}\right)$ is a hereditary cotorsion theory.

Proof. Let $A \in \mathscr{S} \mathscr{M} \mathscr{F}$ and $B \in \mathscr{S} \mathscr{M} \mathscr{F}^{\perp}$. Consider the short exact sequence $0 \rightarrow C \rightarrow$ $P \rightarrow A \rightarrow 0$ with $P$ projective. Then $\operatorname{Ext}_{R}^{2}(A, B) \cong \operatorname{Ext}_{R}^{1}(C, B)=0$ by Proposition 2.6. Let $0 \rightarrow B \rightarrow E \rightarrow D \rightarrow 0$ be an exact sequence with $E$ injective. Then $\operatorname{Ext}_{R}^{1}(A, D) \cong$ $\operatorname{Ext}_{R}^{2}(A, B)=0$, and so $D \in \mathscr{S} \mathscr{M} \mathscr{F}^{\perp}$. Now let $G \in{ }^{\perp}\left(\mathscr{S} \mathscr{M} \mathscr{F}^{\perp}\right)$, then $\operatorname{Ext}_{R}^{2}(G, B) \cong$ $\operatorname{Ext}_{R}^{1}(G, D)=0$. Therefore $\operatorname{Ext}_{R}^{i}(G, B)=0$ for any $i \geq 1$ by induction. Since max-flat modules are contained in $\mathscr{S} \mathscr{M} \mathscr{F}^{\perp}$, $\operatorname{Ext}_{R}^{i}(G, F)=0$ for any max-flat right $R$-module $F$ and $i \geq 1$, so $G \in \mathscr{S} \mathscr{M} \mathscr{F}$. Hence $\left(\mathscr{S} \mathscr{M} \mathscr{F}, \mathscr{S} \mathscr{M} \mathscr{F}^{\perp}\right)=\left({ }^{\perp}\left(\mathscr{S} \mathscr{M} \mathscr{F}^{\perp}\right), \mathscr{S} \mathscr{M} \mathscr{F}^{\perp}\right)$ is a cotorsion theory. Let $0 \rightarrow K \rightarrow L \rightarrow M \rightarrow 0$ be an exact sequence with $L, M \in \mathscr{S} \mathscr{M} \mathscr{F}$. Take $N \in \mathscr{S} \mathscr{M} \mathscr{F}^{\perp}$. Then the sequence $0=\operatorname{Ext}_{R}^{1}(L, N) \rightarrow \operatorname{Ext}_{R}^{1}(K, N) \rightarrow \operatorname{Ext}_{R}^{2}(M, N)=0$ is exact, whence $\operatorname{Ext}_{R}^{1}(K, N)=0$ for any $N \in \mathscr{S} \mathscr{M} \mathscr{F}^{\perp}$. Thus $K \in \mathscr{S} \mathscr{M} \mathscr{F}$.

Now we have the following characterizations of modules with finite $M F$-projective dimension. 
Proposition 3.6. Let $R$ be a ring, $n$ a nonnegative integer and $A$ a right $R$-module. The following are equivalent.

(1) $\operatorname{mfpd}(A) \leq n$.

(2) $\operatorname{Ext}_{R}^{n+i}(A, B)=0$ for any right $R$-module $B \in \mathscr{S} \mathscr{M} \mathscr{F}^{\perp}$ and $i \geq 1$.

(3) $\operatorname{Ext}_{R}^{n+1}(A, B)=0$ for any right $R$-module $B \in \mathscr{S} \mathscr{M} \mathscr{F}^{\perp}$.

(4) If $0 \rightarrow C \rightarrow B_{n-1} \rightarrow \ldots \rightarrow B_{1} \rightarrow B_{0} \rightarrow A \rightarrow 0$ is exact with each $B_{i}$ projective, then $C$ is $S M F$-projective.

(5) There exists an exact sequence $0 \rightarrow B_{n} \rightarrow B_{n-1} \rightarrow \ldots \rightarrow B_{1} \rightarrow B_{0} \rightarrow A \rightarrow 0$ with each $B_{i} S M F$-projective.

Proof. $(2) \Rightarrow(1)$ and $(4) \Rightarrow(5)$ are trivial.

$(1) \Rightarrow(4)$ Let $0 \rightarrow C \rightarrow B_{n-1} \rightarrow \ldots \rightarrow B_{1} \rightarrow B_{0} \rightarrow A \rightarrow 0$ be an exact sequence with each $B_{i}$ projective. Then $\operatorname{Ext}_{R}^{i}(C, B) \cong \operatorname{Ext}_{R}^{n+i}(A, B)=0$ for any max-flat right $R$-module $B$ and $i \geq 1$ by (1). So $C$ is $S M F$-projective by definition.

(4) $\Rightarrow$ (3) Let $0 \rightarrow C \rightarrow B_{n-1} \rightarrow \ldots \rightarrow B_{1} \rightarrow B_{0} \rightarrow A \rightarrow 0$ be an exact sequence with each $B_{i}$ projective. Then $\operatorname{Ext}_{R}^{n+1}(A, B) \cong \operatorname{Ext}_{R}^{1}(C, B)=0$ for any $B \in \mathscr{S} \mathscr{M} \mathscr{F}^{\perp}$.

(3) $\Rightarrow(2)$ For any $B \in \mathscr{S} \mathscr{M} \mathscr{F}^{\perp}$, consider the short exact sequence $0 \rightarrow B \rightarrow E \rightarrow C \rightarrow$ 0 with $E$ injective. Then the sequence $\operatorname{Ext}_{R}^{n+1}(A, C) \rightarrow \operatorname{Ext}_{R}^{n+2}(A, B) \rightarrow \operatorname{Ext}_{R}^{n+2}(A, E)=0$ is exact. Since $E \in \mathscr{S} \mathscr{M}_{\mathscr{F}}^{\perp}, C \in \mathscr{S} \mathscr{M} \mathscr{F}^{\perp}$ by Lemma 3.5 , and so $\operatorname{Ext}_{R}^{n+1}(A, C)=0$ by (3). Therefore $\operatorname{Ext}_{R}^{n+2}(A, B)=0$, and (2) holds by induction.

$(5) \Rightarrow(1)$ Let $B$ be a max-flat right $R$-module and $K_{1}=\operatorname{ker}\left(B_{0} \rightarrow A\right), K_{i}=$ $\operatorname{ker}\left(B_{i-1} \rightarrow B_{i-2}\right)$ for $i \geq 2$. Since each $B_{i}$ is $S M F$-projective, we get that $\operatorname{Ext}_{R}^{n+i}(A, B) \cong$ $\operatorname{Ext}_{R}^{n+i-1}\left(K_{1}, B\right) \cong \ldots \cong \operatorname{Ext}_{R}^{i}\left(B_{n}, B\right)=0$ for any $i \geq 1$. So, $m f p d(A) \leq n$.

Now we set out to investigate how MF-projective dimension behave in short exact sequences. It is easy to check the following result.

Proposition 3.7. Let $R$ be a ring, $0 \rightarrow A \rightarrow B \rightarrow C \rightarrow 0$ an exact sequence of right $R$-modules. If two of $m f p d(A), m f p d(B), m f p d(C)$ are finite, so is the third. Moreover:

(1) $m f p d(B) \leq \sup \{m f p d(A), m f p d(C)\}$;

(2) $m f p d(A) \leq \sup \{m f p d(B), m f p d(C)-1\}$;

(3) $\operatorname{mfpd}(C) \leq \sup \{m f p d(B), m f p d(A)+1\}$.

(4) If $0<m f p d(A)<\infty$ and $B$ is $S M F$-projective, then $\operatorname{mfpd}(C)=\operatorname{mfpd}(A)+1$.

Now we are in the position of characterizing the rings with finite $M F$-projective dimension.

Theorem 3.8. Let $R$ be a ring, $n$ a nonnegative integer. The following are equivalent.

(1) $\operatorname{rmfpD}(R) \leq n$.

(2) $\operatorname{mfpd}(A) \leq n$ for any cyclic right $R$-module $A$.

(3) $i d(A) \leq n$ for all max-flat right $R$-modules $A$.

(4) $i d(A) \leq n$ for all right $R$-modules $A \in \mathscr{S} \mathscr{M}^{\perp}$.

Proof. (1) $\Rightarrow(2)$ and (4) $\Rightarrow(3)$ are trivial.

$(3) \Rightarrow(1)$ Let $A$ be any right $R$-module and $B$ a max-flat right $R$-module. Since $i d(B) \leq n, \operatorname{Ext}_{R}^{n+i}(A, B)=0$ for any $i \geq 1$. Hence $m f p d(A) \leq n$ by definition.

$(2) \Rightarrow(4)$ Let $A \in \mathscr{S} \mathscr{M} \mathscr{F}^{\perp}$ and $I$ be a right ideal of $R$. So $m f p d(R / I) \leq n$, whence by Proposition 3.6, $\operatorname{Ext}_{R}^{n+1}(R / I, A)=0$ for any $n \geq 0$. Thus $i d(A) \leq n$.

We show in Proposition 2.11 that $R$ is a $Q F$ ring if and only if every right $R$-module is $(S) M F$-projective. The following corollary gives a new characterization of $Q F$ rings by using the $M F$-projective modules. 
Corollary 3.9. Let $R$ be a ring. The following are equivalent.

(1) $R$ is a $Q F$-ring.

(2) $\operatorname{rmfpD}(R)=0$.

(3) Every cyclic right $R$-module is $S M F$-projective.

(4) Every max-flat right $R$-module is injective.

(5) Every quotient module of an injective right $R$-module is $M F$-projective.

Moreover, if $R$ is a right max-coherent right $C$-ring, then the above conditions are equivalent to:

(6) Every simple right $R$-module is $M F$-projective.

(7) $R$ is a right max-injective ring.

Proof. By Proposition 2.11 and Theorem 3.8, it is enough to show that $(5) \Rightarrow(4)$ and $(6) \Rightarrow(7) \Rightarrow(1)$.

$(5) \Rightarrow(4)$ For any max-flat right $R$-module $F$, there exists an exact sequence $0 \rightarrow F \rightarrow$ $E \rightarrow B \rightarrow 0$ with $E$ injective. Then $B$ is $M F$-projective by (5), and so $\operatorname{Ext}_{R}^{1}(B, F)=0$. Thus the above short exact sequence splits, which implies that $F$ is injective.

(6) $\Rightarrow(7)$ Since every simple right $R$-module is $M F$-projective, every max-flat right $R$-module is max-injective by Lemma 2.12 . This means that every flat right $R$-module is max-injective. Thus $R$ is a right max-injective ring.

$(7) \Rightarrow(1)$ Let $A$ be a projective right $R$-module. So $A$ is a direct summand of a free module $R^{(I)}$, for some index set $I$. Since $R$ is a right max-injective ring, $R^{(I)}$ is a maxinjective right $R$-module by [27, Proposition 2.4(2)], and so $A$ is max-injective. Also since $R$ is a right $C$-ring, $A$ is injective by [24, Lemma 4]. Thus $R$ is a $Q F$ ring.

Next, we introduce and study $M F$-hereditary rings. But, first, recall that a ring $R$ is called right hereditary if every right ideal is projective. It is known that a ring $R$ is right hereditary if and only if every submodule of a projective right $R$-module is projective (see [23, Theorem 4.23]). We shall say that a ring $R$ is right $M F$-hereditary if every right ideal of $R$ is $M F$-projective. The next theorem gives some characterizations of such rings.

Corollary 3.10. Let $R$ be a ring. The following are equivalent.

(1) $\operatorname{rmfpD}(R) \leq 1$.

(2) $i d(A) \leq 1$ for all max-flat right $R$-modules $A$.

(3) $R$ is right $M F$-hereditary.

(4) Every submodule of any MF-projective right $R$-module is $M F$-projective.

(5) Every submodule of any projective right $R$-module is $M F$-projective.

(6) Every submodule of any free right $R$-module is $M F$-projective.

Proof. $(4) \Rightarrow(5) \Rightarrow(6) \Rightarrow(3)$ are trivial.

(1) $\Leftrightarrow(2)$ follows by Theorem 3.8 .

$(2) \Rightarrow(4)$ Let $B$ be a submodule of an $M F$-projective right $R$-module $A$. Consider the short exact sequence $0 \rightarrow B \rightarrow A \rightarrow A / B \rightarrow 0$. Then for any max-flat right $R$ module $F$, we get an exact sequence $0=\operatorname{Ext}_{R}^{1}(A, F) \rightarrow \operatorname{Ext}_{R}^{1}(B, F) \rightarrow \operatorname{Ext}_{R}^{2}(A / B, F)$. Since $i d(F) \leq 1$, it follows that $\operatorname{Ext}_{R}^{2}(A / B, F)=0$. So $\operatorname{Ext}_{R}^{1}(B, F)=0$, whence $B$ is $M F$-projective.

$(3) \Rightarrow(2)$ Let $F$ be a max-flat right $R$-module and $I$ a right ideal of $R$. Consider the short exact sequence $0 \rightarrow I \rightarrow R \rightarrow R / I \rightarrow 0$. Since $I$ is $M F$-projective, we have

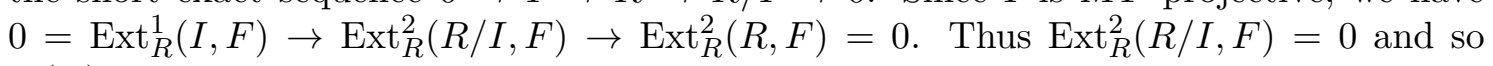
$i d(F) \leq 1$.

It is obvious that every right hereditary ring is right $M F$-hereditary. The following is an example of a right non-hereditary ring $R$ such that every right ideal is $M F$-projective. 
Example 3.11. Let $R$ be a non-semisimple $Q F$ ring. Since by Proposition 2.11, every right $R$-module is $M F$-projective over a $Q F$ ring $R, R$ is a right $M F$-hereditary ring. But $R$ is a non-hereditary ring, otherwise it would be semisimple.

Now we discuss the relations between the class of right $M F$-hereditary rings and the well-known class of right hereditary rings.

Corollary 3.12. Consider the following statements for a ring $R$ :

(1) $R$ is right $M F$-hereditary and left max-hereditary.

(2) $R$ is right $M F$-hereditary and every $M F$-projective right $R$-module is projective.

(3) $R$ is right hereditary.

Then $(1) \Rightarrow(2) \Leftrightarrow(3)$.

Proof. (2) $\Rightarrow(3)$ is clear.

$(1) \Rightarrow(2)$ Let $A$ be an $M F$-projective right $R$-module. Since $R$ is left max-hereditary, $A$ is projective by Proposition 2.5 .

$(3) \Rightarrow(2)$ Assume $R$ is right hereditary. Let $A$ be an $M F$-projective right $R$-module. Consider the exact sequence $0 \rightarrow B \rightarrow F \rightarrow A \rightarrow 0$ with $F$ projective. Since $R$ is right hereditary, $B$ is projective and so $\operatorname{Ext}_{R}^{1}(A, B)=0$. This implies that $0 \rightarrow B \rightarrow F \rightarrow A \rightarrow 0$ splits, whence $A$ is projective.

\section{Max-flat preenvelopes which are epimorphisms}

Recall by [27, Theorem 2.5] that over a left max-coherent $\operatorname{ring} R$, every right $R$-module has a max-flat preenvelope. It is shown that over a left max-coherent ring $R$, every right $R$-module has a monic max-flat preenvelope if and only if $R$ is a left max-injective ring ([27, Theorem 2.11]). It is well known that every right $R$-module has an epic flat envelope if and only if $R$ is a left semihereditary ring ([21, Corollary 4.3]). In this section, we consider when every $R$-module has an epic max-flat preenvelope.

The following lemma gives a characterization of max-flat modules in terms of s-purity.

Lemma 4.1. A right $R$-module $A$ is max-flat if and only if any short exact sequence ending with $A$ is s-pure.

Proof. Let $0 \rightarrow C \rightarrow B \rightarrow A \rightarrow 0$ be an exact sequence. Since $A$ is max-flat, for any maximal left ideal $I$ of $R$, we have the exact sequence $0=\operatorname{Tor}_{1}^{R}(A, R / I) \rightarrow C \otimes R / I \rightarrow B \otimes$ $R / I \rightarrow A \otimes R / I \rightarrow 0$. So the exact sequence $0 \rightarrow C \rightarrow B \rightarrow A \rightarrow 0$ is s-pure. Conversely, let $0 \rightarrow B \rightarrow F \rightarrow A \rightarrow 0$ be an s-pure exact sequence with $F$ projective. For any maximal left ideal $I$ of $R$, we have the exact sequence $0=\operatorname{Tor}_{1}^{R}(F, R / I) \rightarrow \operatorname{Tor}_{1}^{R}(A, R / I) \rightarrow$ $B \otimes R / I \rightarrow F \otimes R / I$. Since $B \otimes R / I \rightarrow F \otimes R / I$ is monic, $\operatorname{Tor}_{1}^{R}(A, R / I)=0$. Hence, $A$ is max-flat.

Unlike the generation of pure submodules the notions of s-pure and neat submodules are not only inequivalent they are also incomparable. Recently, the commutative rings for which the notions of s-pure and neat submodules are equivalent are completely characterized in [19, Theorem 3.7]. These are exactly the commutative rings whose maximal ideals are finitely generated and locally principal. A right module $A$ is called neat-flat if for any epimorphism $f: B \rightarrow A$, the induced map $\operatorname{Hom}(S, B) \rightarrow \operatorname{Hom}(S, A)$ is epic for any simple right module $S$, equivalently any short exact sequence ending with $A$ is neat-exact (see [3]). Together with Lemma 4.1 and [3, Lemma 2.3.], we obtain the following. 
Corollary 4.2. Let $R$ be a commutative ring whose maximal ideals are finitely generated and locally principal and let $A$ be an $R$-module. Then the following are equivalent.

(1) A is max-flat.

(2) A is neat-flat.

(3) $A$ is simple projective, i.e. for any simple $R$-module $S$, every homomorphism $f: S \rightarrow A$ factors through a finitely generated free $R$-module $F$.

If $R$ is a left max-hereditary ring, then every MF-projective right module is projective by Proposition 2.5. Now for the converse, we have the following characterizations of max-hereditary rings.

Theorem 4.3. Let $R$ be a commutative ring whose maximal ideals are finitely generated and locally principal. The following are equivalent.

(1) $R$ is max-hereditary.

(2) Every MF-projective $R$-module is projective.

(3) Every $M F$-projective $R$-module is flat.

(4) Every finitely presented $M F$-projective $R$-module is projective.

(5) Every simple R-module has an epic projective preenvelope.

(6) Every simple $R$-module has an epic max-flat preenvelope.

(7) Every $R$-module has an epic max-flat preenvelope.

(8) Every submodule of a max-flat $R$-module is max-flat.

Proof. (1) $\Rightarrow(2)$ is by Proposition 2.5.

$(2) \Rightarrow(3)$ and $(7) \Rightarrow(6)$ are clear.

$(6) \Rightarrow(5) \Rightarrow(8)$ is by Corollary 4.2 and [18, Theorem 3.7].

$(3) \Rightarrow(4)$ Let $A$ be a finitely presented $M F$-projective $R$-module. Then $A$ is flat by (3), and so is projective since $A$ is finitely presented.

$(4) \Rightarrow(5)$ Let $S$ be a simple $R$-module. Since $R$ is max-coherent, $S$ has a max-flat preenvelope $\psi: S \rightarrow F$ with $F$ max-flat. So $\psi$ factors through a finitely generated free module $P$ by Corollary 4.2. This means that there exist homomorphisms $f: S \rightarrow P$ and $g: P \rightarrow F$ such that $g f=\psi$. Let $B=\operatorname{Im}(f), \beta: S \rightarrow B$ and $A=P / B$. Now, we claim that the inclusion map $i: B \rightarrow P$ is a max-flat preenvelope of $B$. Let $h: B \rightarrow M$ be a homomorphism with $M$ max-flat. Then there exists a homomorphism $\phi: F \rightarrow M$ such that $\phi g f=\phi g i \beta=h \beta$. Since $\beta$ is epic, $h=(\phi g) i$. This proves our claim, whence $A$ is $M I$-flat by [27, Proposition 3.7(1)]. Since $A$ is finitely presented, $A$ is $M F$-projective by Proposition 2.9(2), and so is projective by the hypothesis. Thus the splitting of $0 \rightarrow B \rightarrow P \rightarrow A \rightarrow 0$ says that $B$ is projective. Hence $S \rightarrow B$ is a projective preenvelope which is an epimorphism.

$(8) \Rightarrow(1)$ Let $B$ be a factor of a max-injective $R$-module $A$. Then the exact sequence $0 \rightarrow C \rightarrow A \rightarrow B \rightarrow 0$ induces the exactness of $0 \rightarrow B^{+} \rightarrow A^{+} \rightarrow C^{+} \rightarrow 0$. Since $A^{+}$is max-flat by [27, Theorem 2.3], $B^{+}$is max-flat by (8) and so $B$ is max-injective. Hence by [1, Proposition 1.2], $R$ is max-hereditary.

$(8) \Rightarrow(7)$ For any $R$-module $A$, there is a max-flat preenvelope $f: A \rightarrow B$. Note that $\operatorname{Im}(f)$ is max-flat by (8), so $A \rightarrow \operatorname{Im}(f)$ is an epic max-flat preenvelope.

$R$ is called a right $P S$ ring [20] if every simple right ideal is projective. It is shown that every submodule of any neat-flat right $R$-module is neat-flat if and only if $R$ is a right PS ring ([2, Theorem 5.3]). As a consequence of Corollary 4.2 and Theorem 4.3, we obtain a new characterization of max-hereditary rings.

Corollary 4.4. Let $R$ be a commutative ring whose maximal ideals are finitely generated and locally principal. The following are equivalent.

(1) $R$ is a max-hereditary ring.

(2) $R$ is a PS ring. 


\section{References}

[1] Y. Alagöz, On m-injective and m-projective modules, Math. Sci. Appl. E-Notes, 8, 46-50, 2020.

[2] E. Büyükaşık and Y. Durğun, Absolutely s-pure modules and neat-flat modules, Comm. Algebra, 43 (2), 384-399, 2015.

[3] E. Büyükaşık and Y. Durğun, Neat-flat modules. Comm. Algebra 44 (1), 416-428, 2016.

[4] J. Clark, C. Lomp, N. Vanaja and R. Wisbauer, Lifting modules, Frontiers in Mathematics, Birkhäuser Verlag, Basel, 2006.

[5] I. Crivei, s-pure submodules, Int. J. Math. Math. Sci. 4, 491-497, 2005.

[6] S. Crivei, Neat and coneat submodules of modules over commutative rings, Bull. Aust. Math. Soc. 89 (2), 343-352, 2014.

[7] Y. Durğun, On some generalizations of closed submodules, Bull. Korean Math. Soc. 52 (5), 1549-1557, 2015.

[8] E.E. Enochs and O.M.G Jenda, Relative homological algebra, de Gruyter, Berlin, 2000.

[9] E.E. Enochs, O.M.G. Jenda and J.A. Lopez-Ramos, The existence of Gorenstein flat covers, Math. Scand. 94 (1), 46-62, 2004.

[10] C. Faith, Algebra. II, Springer-Verlag, Berlin-New York, 1976. Ring theory, Grundlehren der Mathematischen Wissenschaften, No. 191.

[11] X. Fu, H. Zhu and N. Ding, On Copure Projective Modules and Copure Projective Dimensions, Comm. Algebra, 40 (1), 343-359, 2012.

[12] L. Fuchs, Neat submodules over integral domains, Period. Math. Hungar. 64 (2), 131-143, 2012.

[13] M.F. Hamid, Coneat injective modules, Missouri J. Math. Sci. 31 (2), 201-211, 2019.

[14] K. Honda, Realism in the theory of abelian groups I, Comment. Math. Univ. St. Pauli 5, 37-75, 1956.

[15] H. Holm and P. Jorgensen, Covers, precovers, and purity, Illinois J. Math. 52 (2), 691-703, 2008.

[16] C.U. Jensen and D. Simon, Purity and generalized chain conditions, J. Pure Appl. Algebra 14, 297-305, 1979.

[17] T.Y. Lam, Lectures on modules and rings, Springer-Verlag, New York, 1999.

[18] L. Mao, When does every simple module have a projective envelope?, Comm. Algebra, 35 (5), 1505-1516, 2007.

[19] E. Mermut and Z. Türkoğlu, Neat submodules over commutative rings, Comm. Algebra, 48 (3), 1231-1248, 2020.

[20] W.K. Nicholson and J.F. Watters, Rings with projective socle, Proc. Amer. Math. Soc. 102, 443-450, 1988.

[21] J. Rada and M. Saorin, Rings characterized by (pre)envelopes and (pre)covers of their modules, Comm. Algebra, 26 (3), 899-912, 1998.

[22] V.S. Ramamurthi, On the injectivity and flatness of certain cyclic modules, Proc. Amer. Math. Soc. 48, 21-25, 1975.

[23] J.J. Rotman, An Introduction to Homological Algebra, in Pure Appl.Math., Vol. 85, Academic Press, New York, 1979.

[24] P.F. Smith, Injective modules and prime ideals. Comm. Algebra, 9 (9), 989-999, 1981.

[25] M.Y. Wang, Frobenius structure in algebra (chinese). Science Press, Beijing, 2005.

[26] M.Y. Wang and G. Zhao, On maximal injectivity, Acta Math. Sin. (Engl. Ser.) 21 (6), 1451-1458, 2005.

[27] Y. Xiang, Max-injective, max-flat modules and max-coherent rings, Bull. Korean Math. Soc. 47 (3), 611-622, 2010. 